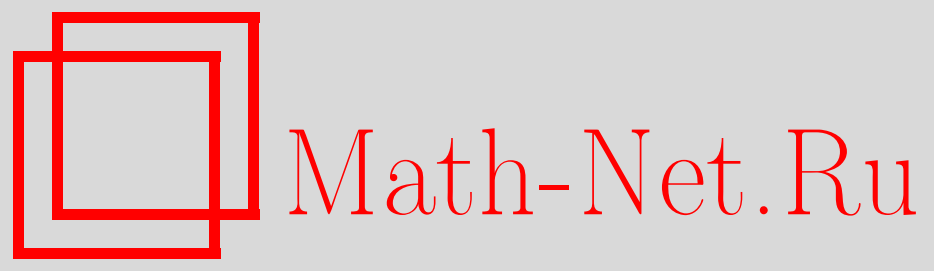

Р. Т. Сибатов, В. В. Светухин, Субдиффузионная кинетика роста и разрушения нанопреципитатов в твердых растворах, ТMФ, 2015, том 183, номер 3, 460-476

DOI: https://doi.org/10.4213/tmf8832

Использование Общероссийского математического портала Math-Net.Ru подразумевает, что вы прочитали и согласны с пользовательским соглашением http://www.mathnet.ru/rus/agreement

Параметры загрузки:

IP: 34.227 .88 .159

26 апреля 2023 г., 18:18:41

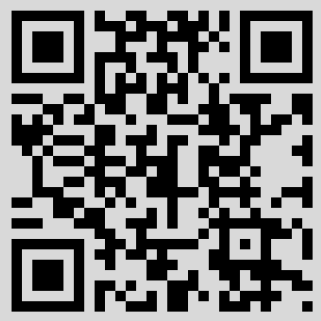




\title{
СУБДИФФУЗИОННАЯ КИНЕТИКА РОСТА И РАЗРУШЕНИЯ НАНОПРЕЦИПИТАТОВ В ТВЕРДЫХ РАСТВОРАХ
}

\begin{abstract}
На основе дробно-дифференциальных обобщений моделей преципитации Хэма и Аарона-Котлера исследуется кинетика субдиффузионно-ограниченного роста и растворения выделений новой фазы. Получены зависимости числа примесей и размеров выделения новой фазы от времени. Решения согласуются с результатами моделирования методом Монте-Карло.
\end{abstract}

Ключевые слова: субдиффузия, нанопреципитат, дробная производная, пересыщенный твердый раствор, метод Монте-Карло.

DOI: $10.4213 / \operatorname{tmf} 8832$

\section{1. ВВЕДЕНИЕ}

Как известно, для описания распада пересыщенных твердых растворов (преципитации) на стадии роста выделений новой фазы с успехом применяются диффузионные модели [1]-[10], в основе которых лежит представление о нормальной диффузии примесей. Однако ряд экспериментальных фактов свидетельствует об аномальной диффузии (преимущественно субдиффузии) примесей и дефектов в различных материалах [11]-[15]. Ее принято характеризовать с помощью закона расширения диффузионного пакета $\Delta(t) \propto t^{\alpha / 2}$. В основе классической диффузии $(\alpha=1)$ лежит гауссова статистика и второй закон Фика, которые приводят к зависимости $\Delta(t)=\sqrt{\left\langle x^{2}(t)\right\rangle} \propto t^{1 / 2}$ [16]. В случае $0<\alpha<1$ ширина диффузионного пакета увеличивается со временем медленнее (субдиффузия), а в случае $\alpha>1$ - быстрее (супердиффузия), чем в нормальном случае. Математическую основу аномальной автомодельной (самоподобной) диффузии образуют уравнения в дробных производных [14], [17], [18].

Существующие диффузионные модели преципитации недостаточно полно отражают свойства явлений переноса, протекающих в реальных материалах, для которых наличие беспорядка приводит к существенному изменению типа диффузии [12], [13].

\footnotetext{
*Ульяновский государственный университет, лаборатория моделирования диффузионных процессов, Ульяновск, Россия. E-mail: ren_sib@bk.ru
} 
Применение уравнений диффузии в дробных производных позволяет в значительной мере упростить рассмотрение таких сложных явлений, как диффузия в неоднородных средах, диффузия по границам зерен, дислокациям и др. [14], [18].

В настоящей работе исследуется субдиффузионно-ограниченная кинетика роста и распада выделений новой фазы с помощью дробно-дифференциальных обобщений классических моделей роста и растворения преципитатов в твердых растворах [2], [3], [7]. В качестве инструмента описания аномальной диффузии в неупорядоченных средах применяется теория дисперсионного транспорта, развитая в работах [18]-[21]. Аналитические решения сравниваются с результатами моделирования процесса методом Монте-Карло.

\section{2. СЛУЧАЙНЫЕ БЛУЖДАНИЯ С ЛОКАЛИЗАЦИЕЙ ПРИМЕСЕЙ}

Для описания эредитарной диффузии частиц (диффузии с задержкой, связанной с временами локализации) часто применяется [14], [22] интегро-дифференциальное уравнение вида

$$
\frac{\partial \rho(\mathbf{r}, t)}{\partial t}=C \int_{0}^{t} d \tau Q(t-\tau) \nabla^{2} \rho(\mathbf{r}, t)
$$

где $Q(t)$ - ядро памяти, а под $\rho$ подразумевается суммарная концентрация частиц: локализованных и подвижных. Закон Фика $\mathbf{j}(\mathbf{r}, t)=-D \nabla \rho(\mathbf{r}, t)$ в этом случае неприменим, необходимо прибегать к обобщенному закону Фика [23]. Уравнение (1) можно получить на основе модели случайных блужданий с непрерывным временем (СБНВ, в англоязычной литературе CTRW - Continuous time random walk) [24]-[29]. Подразумевается, что частицы меняют свои позиции скачком в случайные моменты времени, при этом интервалы времени между прыжками (времена ожсдания)независимые одинаково распределенные случайные величины $\tau$.

Интегральное уравнение модели СБНВ для плотности распределения координаты $x$ блуждающей частицы в момент времени $t$ имеет вид [29]

$$
\rho(\mathbf{r}, t)=\Psi(t) \delta(\mathbf{r})+\int_{0}^{t} d t^{\prime} \psi\left(t^{\prime}\right) \int d \mathbf{r}^{\prime} w\left(\mathbf{r}^{\prime}\right) \rho\left(\mathbf{r}-\mathbf{r}^{\prime}, t-t^{\prime}\right),
$$

где $\psi(t)$ - плотность распределения времен пребывания частиц в локализованном состоянии, $\Psi(t)=\int_{t}^{\infty} d \tau \psi(\tau)$ - дополнительная функция распределения, $w(\mathbf{r})-$ плотность распределения элементарного смещения. Подразумевается, что в момент $t=0$ частица попадает в локализованное состояние в начале координат.

Преобразование Фурье-Лапласа уравнения (2) приводит к алгебраическому соотношению, из которого можно найти образ функции $\rho(\mathbf{r}, t)$ (формула Монтролла-Вейса [30]):

$$
\widetilde{\hat{\rho}}(\mathbf{k}, s)=\frac{\widetilde{\Psi}(s)}{1-\hat{w}(\mathbf{k}) \tilde{\psi}(s)}, \quad \tilde{\hat{\rho}}(\mathbf{k}, s)=\int_{0}^{t} d t \int d \mathbf{r} e^{i \mathbf{k r}-s t} \rho(\mathbf{r}, t),
$$

где $\hat{w}(\mathbf{k})$ - преобразование Фурье функции $w(\mathbf{r}), \widetilde{\Psi}(s)$ - образ Лапласа функции $\Psi(t)$, связанный с образом Лапласа плотности распределения соотношениями

$$
\widetilde{\Psi}(s)=\frac{1-\tilde{\psi}(s)}{s}, \quad \tilde{\psi}(s)=1-s \widetilde{\Psi}(s) .
$$


Подстановка разложения при малых $|\mathbf{k}|$ характеристической функции плотности распределения смещений с нулевым средним и конечной дисперсией $\hat{w}(\mathbf{k}) \sim 1-C|\mathbf{k}|^{2}$ и выражения (4) в уравнение Монтролла-Вейса (3) приводит к уравнению, которое может быть записано в следующих двух эквивалентных видах:

$$
\begin{aligned}
& {\left[s+C \frac{\tilde{\psi}(s)}{\widetilde{\Psi}(s)}|\mathbf{k}|^{2}\right] \tilde{\rho}(k, s)=1,} \\
& {\left[\frac{s \widetilde{\Psi}(s)}{\tilde{\psi}(s)}+C|\mathbf{k}|^{2}\right] \tilde{\rho}(k, s)=\frac{\widetilde{\Psi}(s)}{\tilde{\psi}(s)} .}
\end{aligned}
$$

Обратное преобразование Фурье-Лапласа первого из них приводит к эредитарному уравнению диффузии (1), где образ ядра памяти имеет вид $\widetilde{Q}(s)=\widetilde{\psi}(s) / \widetilde{\Psi}(s)$. Для уравнения (6) можно записать

$$
\frac{\partial}{\partial t} \int_{0}^{t} d \tau \rho(\mathbf{r}, \tau) \Phi(t-\tau)-C \nabla^{2} \rho(\mathbf{r}, t)=\delta(\mathbf{r}) \Phi(t),
$$

где $\Phi(t)$ - оригинал для образа Лапласа $\widetilde{\Phi}(s)=\widetilde{\Psi}(s) / \tilde{\psi}(s)$.

Можно показать [17], что требование автомодельности (самоподобия) функций Грина уравнения (1) приводит к дробно-дифференциальному уравнению диффузии

$$
\frac{\partial \rho(\mathbf{r}, t)}{\partial t}=C_{\alpha} \nabla^{2}{ }_{0} \mathbf{D}_{t}^{1-\alpha} \rho(\mathbf{r}, t) .
$$

Здесь

$$
{ }_{0} \mathrm{D}_{t}^{\alpha} \rho(\mathbf{r}, t)=\frac{1}{\Gamma(1-\alpha)} \frac{\partial}{\partial t} \int_{0}^{t} d \tau \frac{\rho(\mathbf{r}, \tau)}{(t-\tau)^{\alpha}}
$$

есть дробная производная Римана-Лиувилля [31]-[33]. Порядок производной определяется дисперсионным параметром $0<\alpha \leqslant 1$, который можно найти из эксперимента. Фундаментальные решения этого уравнения приведены в работах [17], [31]. Дробно-дифференциальное уравнение (8) можно вывести как асимптотику уравнения (1) в случае степенного распределения времен локализации:

$$
\Psi(t)=P\{\tau>t\} \propto t^{-\alpha}, \quad \alpha>0, \quad t \rightarrow \infty .
$$

Действительно, подстановка выражений для трансформант Лапласа $\tilde{\psi}(s)=1-b_{\alpha} s^{\alpha}$ и $\widetilde{\Psi}(s)=b_{\alpha} s^{\alpha-1}$ в уравнение (5) в асимптотике $s \rightarrow 0$ приводит к выражению

$$
\left[s+C_{\alpha} s^{1-\alpha}|\mathbf{k}|^{2}\right] \tilde{\rho}(k, s)=1, \quad C_{\alpha}=\frac{C}{b_{\alpha}},
$$

обратное преобразование Лапласа для которого дает уравнение (8) с начальным условием $\left.\rho(\mathbf{r}, t)\right|_{t=0}=\delta(\mathbf{r})$.

Отметим, что представление уравнения субдиффузии в виде (8) удобно для записи уравнения непрерывности

$$
\frac{\partial \rho}{\partial t}+\operatorname{div} \mathbf{j}=0
$$




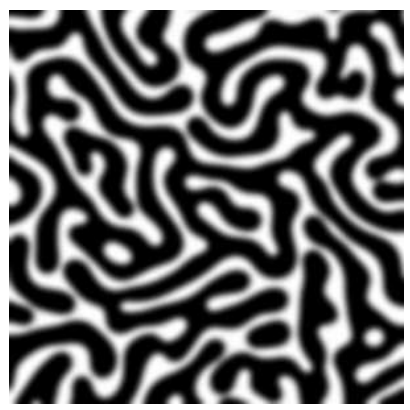

a

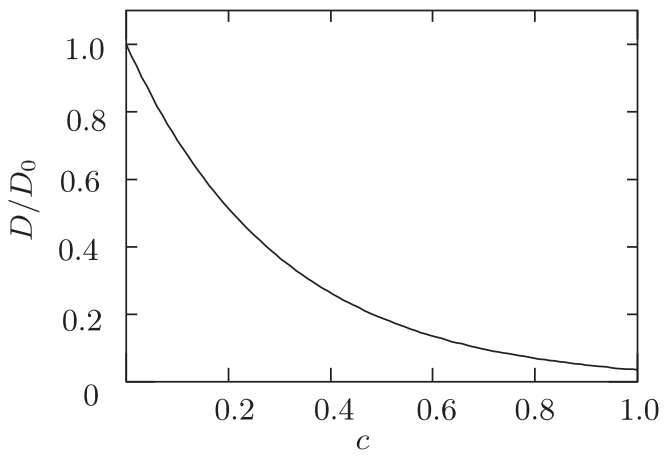

б

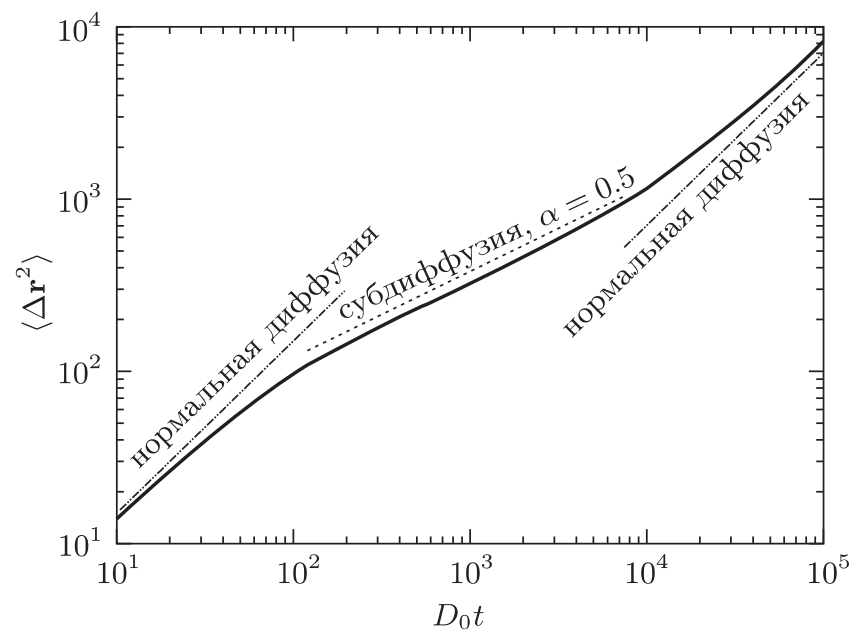

B

Рис. 1. Реализация аномальной диффузии при блуждании частицы на плоской реализации спинодального распада двухкомпонентного раствора: картина спинодального распада, смоделированного методом Монте-Карло в рамках подхода Кана-Хилларда (а); заданная зависимость коэффициента диффузии от состава (б); зависимость среднего квадрата смещения частицы в процессе диффузии, полученная моделированием методом Монте-Карло (в).

в котором плотность тока частиц выражается через концентрацию с помощью обобщенного закона Фика [23]:

$$
\mathbf{j}=-C_{0} \mathrm{D}_{t}^{1-\alpha} \nabla \rho(\mathbf{r}, t)
$$

Уравнения (8) и (12) мы применим для анализа кинетики субдиффузионного роста и растворения частиц новой фазы.

Механизмы аномальной диффузии разнообразны [12], [14], [18]. Нам представляется примечательным тот факт, что субдиффузия может наблюдаться как результат нормальной диффузии в неоднородной среде. На рис. 1 представлены результаты моделирования блуждания частицы (примесного атома или вакансии) на 
плоской реализации спинодального распада двухкомпонентного раствора при равных долях компонентов. Реализация получена методом Монте-Карло в рамках подхода Кана-Хилларда [34]-[36]. При расчете мы полагали, что сечение изотропного рассеяния блуждающей частицы зависит от состава раствора согласно формуле $\sigma=\sigma_{0} e^{-c / c_{0}}$, где $0 \leqslant c \leqslant 1$ есть доля одного из компонентов. Результаты на рис. 1 представлены для случая $c_{0}=0.3$. На зависимости среднего квадрата смещения частицы от времени наблюдается участок субдиффузионного поведения, в асимптотике больших времен диффузия становится нормальной. Такое поведение можно объяснить наличием областей с низким коэффициентом диффузии $(c \approx 1$, см. рис. 1б), которые служат броуновской ловушкой для блуждающих частиц. Времена пребывания в этих областях распределены по усеченному степенному закону. Усечение обуславливает переход к нормальной статистике при $t \rightarrow \infty$. Наличие участка квазинормальной диффузии на малых временах связано с блужданием в однородной области на начальном этапе, влияние неоднородности сказывается на бо́льших масштабах.

Авторы работы [37] использовали так называемый "плавно усеченный степенной закон" в качестве распределения времен ожидания. Представив $\Psi(t)$ в аналогичной форме

$$
\Psi(t) \sim \frac{A t^{-\alpha} e^{-\beta t}}{\Gamma(1-\alpha)},
$$

приходим с помощью уравнения (7) к следующему уравнению диффузии с усеченной дробной производной:

$$
e^{-\beta t}{ }_{0} \mathrm{D}_{t}^{\alpha} e^{\beta t} p(x, t)-C_{\alpha, \beta} \frac{\partial^{2}}{\partial x^{2}} p(x, t)=N \delta(x) \frac{t^{-\alpha} e^{-\beta t}}{\Gamma(1-\alpha)} .
$$

Обобщенный закон Фика в этом случае принимает вид

$$
j(x, t)=-C_{\alpha, \beta} \frac{\partial}{\partial t} e^{-\beta t}{ }_{0} \mathrm{l}_{t}^{\alpha} e^{\beta t} \frac{\partial p(x, t)}{\partial x},
$$

где ${ }_{0} l_{t}^{\alpha}$ - дробный интеграл Римана-Лиувилля. Уравнения (13), (14) могут применяться при описании эффектов, связанных с подавлением субдиффузионного поведения и переходом к гауссовой статистике в асимптотике больших времен.

\section{3. СУБДИФФУЗИОННОЕ ОБОБЩЕНИЕ МОДЕЛИ ХЭМА}

В теории Хэма распада пересыщенного твердого раствора [2] рассматривается процесс роста преципитатов, управляемый нормальной диффузией. Первоначально Хэм рассматривает граничную задачу для уравнения диффузии (8) при условии, что размер выделения новой фазы (преципитата) изменяется очень медленно. Это справедливо, если размер зародыша мал по сравнению с размером ячейки, в которой рассматривается рост, и плотность выделения новой фазы значительно превышает плотность примесей в матрице. Граничные условия, сформулированные для уравнения нормальной диффузии в работе [2], имеют вид

$$
\rho(\mathbf{r}(S), t)=\rho_{\mathrm{I}}, \quad \mathbf{n}_{T} \nabla \rho(\mathbf{r}(T), t)=0 .
$$

Первое условие означает, что плотность раствора на поверхности выделения равна равновесной величине $\rho_{\mathrm{I}}$, имеющей постоянное значение при заданных внешних 
условиях. В работе [2] предполагается, что выделение новой фазы имеет сферическую форму ( $S$ - поверхность сферического кластера), и размер кластера мал по сравнению с расстоянием между кластерами. Зародыши равномерно распределены по образцу, так что можно разбить образец на ячейки и считать, что выделения новой фазы расположены в центре этих ячеек, а границы $T$ представляют собой поверхности ячеек Вигнера-Зейтца. Таким образом, второе граничное условие предполагает, что концентрации раствора по обе стороны от границы приблизительно равны друг другу и не возникает потока частиц из одной ячейки в другую.

Решая граничную задачу при указанных предположениях, Хэм показывает [2], что на границе кластера

$$
\left.\nabla \rho(\mathbf{r}, t)\right|_{r=r_{\mathrm{c}}} \approx \frac{\bar{\rho}(t)}{r_{\mathrm{c}}},
$$

где $\bar{\rho}(t)$ - средняя по ячейке плотность “мономеров" (непреципитировавших частиц). Аналогичное рассмотрение эквивалентной граничной задачи, но уже для дробнодифференциального уравнения (8) привело нас к такому же выражению [38].

Интегрируя уравнение непрерывности (11), где $\mathbf{j}$ определяется согласно обобщенному закону Фика (12), по объему эквивалентной сферы с применением теоремы Гаусса, приходим к следующему соотношению:

$$
\frac{d N}{d t}+\int_{S} d \mathbf{S} C_{\alpha{ }_{0}} \mathrm{D}_{t}^{1-\alpha} \nabla \rho(\mathbf{r}, t)=0 .
$$

Здесь интеграл берется по поверхностям ячейки и кластера, и поток через поверхность ячейки равен нулю (второе граничное условие). Остается только интеграл по поверхности сферического преципитата. Область интегрирования со временем изменяется за счет роста кластера. В уравнении (16) $N$ - число "свободных" (непреципитировавших) частиц в ячейке, которое связано со средней концентрацией соотношением

$$
N=\bar{\rho}(t) \frac{4}{3} \pi r_{\mathrm{s}}^{3}
$$

Ввиду сферической симметрии концентрация частиц на поверхности одинакова. Следовательно, поверхностный интеграл в (16) сводится к умножению подынтегральной функции на площадь сферы, и с использованием (17) мы получаем

$$
\frac{4}{3} \pi r_{\mathrm{s}}^{3} \frac{d \bar{\rho}(t)}{d t}=-4 \pi r_{\mathrm{c}}^{2} C_{\alpha 0} \mathrm{D}_{t}^{1-\alpha} \nabla \rho(\mathbf{r}, t)
$$

Подставляя соотношение (15) в правую часть уравнения (18) приходим к уравнению

$$
r_{\mathrm{s}}^{3} \frac{d \bar{\rho}(t)}{d t}=-3 r_{\mathrm{c}} C_{\alpha 0} \mathrm{D}_{t}^{1-\alpha} \bar{\rho}(t)
$$

Из закона сохранения суммарного числа частиц (свободных и в кластере) имеем

$$
\frac{4 \pi}{3} \rho_{\mathrm{c}}\left[r_{\mathrm{c}}^{3}(t)-r_{\mathrm{c}}^{3}(0]=\frac{4 \pi}{3} r_{\mathrm{s}}^{3}\left[\rho_{0}-\bar{\rho}(t)\right]\right.
$$

Левая часть в этом соотношении представляет собой число частиц, присоединенных кластером за время $t$, выраженное через изменение объема кластера. Здесь $\rho_{\text {с }-}$ средняя концентрация частиц в кластере. Правая часть приближенно (пренебрегаем

5 Теоретическая и математическая физика, т. 183, № 3, 2015 г. 
размером сферического выделения) равна тому же самому числу, но выраженному через изменение плотности "свободных" частиц в матрице.

Из соотношения (20) получаем

$$
r_{\mathrm{c}}(t)=\left[r_{\mathrm{c}}^{3}(0)+\frac{r_{\mathrm{s}}^{3}}{\rho_{\mathrm{c}}}\left(\rho_{0}-\bar{\rho}(t)\right)\right]^{1 / 3} .
$$

Подставляем это выражение в соотношение (19) и приходим к дробно-дифференциальному уравнению, описывающему кинетику роста выделения новой фазы, управляемого субдиффузией:

$$
\frac{d \bar{\rho}(t)}{d t}=-\frac{3 C_{\alpha}}{r_{\mathrm{s}}^{3}}\left[r_{\mathrm{c}}^{3}(0)+\frac{r_{\mathrm{s}}^{3}}{\rho_{\mathrm{c}}}\left(\rho_{0}-\bar{\rho}(t)\right)\right]^{1 / 3}{ }_{0} \mathrm{D}_{t}^{1-\alpha} \bar{\rho}(t) .
$$

Это уравнение можно решить с помощью одного из специально разработанных численных методов для дробно-дифференциальных уравнений [39]-[42]. Асимптотический анализ [38] формулы (22) показал, что при достаточно малых $t$, если положить $\bar{\rho}(t) \approx \rho(0)$, решение можно представить в виде

$$
\bar{\rho}(t) \approx \rho_{0} \exp \left\{-\left(\frac{2 C_{\alpha}(\alpha)}{r_{\mathrm{s}}^{2}}\right)^{3 / 2} \sqrt{\frac{\rho_{0}}{\rho_{\mathrm{c}}}} \frac{t^{3 \alpha / 2}}{[\Gamma(\alpha+1)]^{3 / 2}}\right\} .
$$

Это обобщенная формула Хэма. Следует отметить, что и коэффициент диффузии $C_{\alpha}$ определяется по отличной от классической формуле (подробнее см. работу [17]). При больших $t$ мы имеем $\bar{\rho}(t) \ll \rho_{0}$, и из уравнения $(22)$ получается, что решение выражается через специальную функцию - функцию Миттаг-Леффлера [43]

$$
E_{\alpha}(x)=\sum_{n=0}^{\infty} \frac{x^{n}}{\Gamma(a n+1)}, \quad 0<\alpha<1,
$$

играющую важную роль в дробно-дифференциальном исчислении [31]. Решение задается формулой

$$
\bar{\rho}(t)=\rho_{0} E_{\alpha}\left(-K t^{\alpha}\right) \sim \frac{\rho_{0}}{K} \frac{t^{-\alpha}}{\Gamma(1-\alpha)}, \quad t \gg K^{-1 / \alpha} .
$$

Здесь

$$
K=\frac{3 C_{\alpha}}{r_{\mathrm{s}}^{3}}\left[r_{\mathrm{c}}^{3}(0)+\frac{r_{\mathrm{s}}^{3}}{\rho_{\mathrm{c}}} \rho_{0}\right]^{1 / 3} .
$$

Изменение размеров выделения новой фазы можно рассчитать с помощью соотношения (21). При малых временах приходим к выражению

$$
r_{\mathrm{c}}(t)=\left[r_{\mathrm{c}}^{3}(0)+R^{3}\right]^{1 / 3},
$$

где

$$
R=\sqrt{\frac{2 C_{\alpha}(\alpha)}{\Gamma(\alpha+1)} \frac{\rho_{0}}{\rho_{\mathrm{c}}}} t^{\alpha / 2}
$$




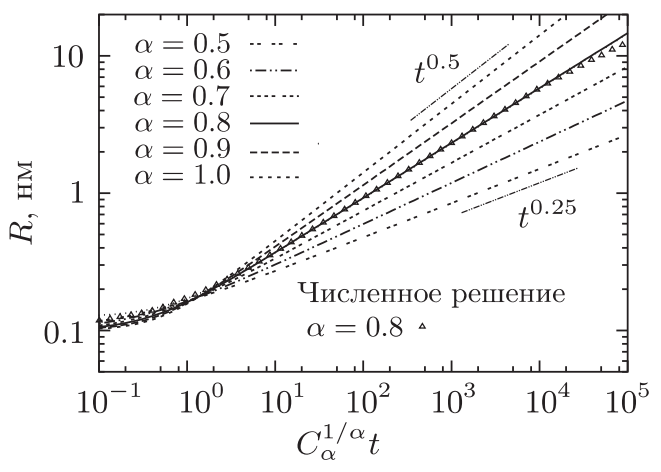

a

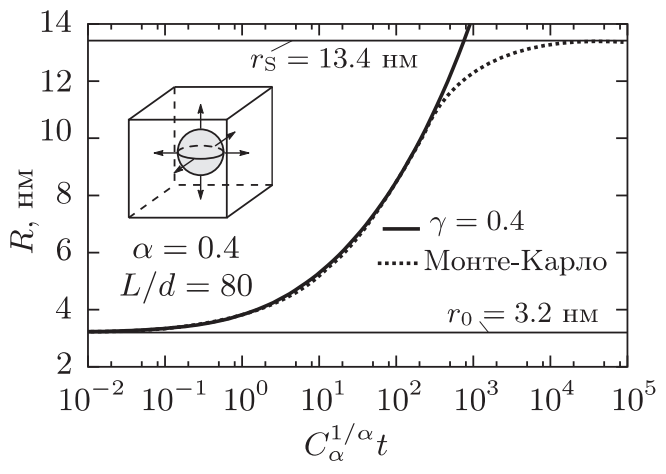

6

Рис. 2. На рисунке "а" изображена зависимость радиуса преципитата от времени для различных значений $\alpha$. Линии - графики функции (25), точки решение $(21)$, где $\bar{\rho}(t)$ найдено с помощью конечно-разностной численной схемы для дробно-дифференциального уравнения (22). Выбранные параметры: $r_{\mathrm{c}}(0)=0.1 \mathrm{Hм}, r_{\mathrm{s}}=100 \mathrm{нм}, \rho_{\mathrm{c}} / \rho_{0}=100$. На рисунке "б" решение $(25)$ приведено в сравнении с результатом моделирования методом Монте-Карло для случая $\alpha=0.4$.

При больших временах

$$
r_{\mathrm{c}}(t)=\left[r_{\mathrm{c}}^{3}(0)+r_{\mathrm{s}}^{3} \frac{\rho_{0}}{\rho_{\mathrm{c}}}\left(1-E_{\alpha}\left(-K t^{\alpha}\right)\right)\right]^{1 / 3} .
$$

На рис. 2 представлено сравнение аналитических решений (25) с численными решениями для уравнений $(21),(22)$ и результатами моделирования методом МонтеКарло (см. рис. 2б), описанного в следующем разделе.

\section{4. МОДЕЛИРОВАНИЕ МЕТОДОМ МОНТЕ-КАРЛО В РАМКАХ ПОДХОДА ХЭМА}

В работе [44] предложен алгоритм Монте-Карло решения интегро-дифференциального уравнения с дробной производной, основанный на применении модели дробного пуассоновского процесса [45]. Этот алгоритм использован для решения уравнения дисперсионного переноса в неупорядоченных полупроводниках. В настоящей работе данный алгоритм применяется для расчета многочастичной задачи субдиффузионного распада твердых растворов на стадии роста частиц новой фазы различной формы. Рост сферических выделений моделировался в работе [38].

Процесс роста частиц новой фазы, ограниченного субдиффузией, моделировался следующим образом. Генерировался ансамбль из $N_{\mathrm{p}}$ частиц, однородно распределенных в кубической ячейке размера $L$, которая в центре содержала зародыш новой фазы. Каждой частице приписывались координаты $x_{i}, y_{i}, z_{i}$ и время пребывания в ловушке $T_{i}$, разыгранное по закону [45] (здесь и ниже равенство $\stackrel{\mathrm{d}}{=}$ означает совпадение распределений)

$$
T \stackrel{\mathrm{d}}{=} \frac{\left|\ln U_{1}\right|^{1 / \alpha}}{\mu^{1 / \alpha}} \frac{\sin \left(\alpha \pi U_{2}\right)\left[\sin \left((1-\alpha) \pi U_{2}\right)\right]^{1 / \alpha-1}}{\left[\sin \left(\pi U_{2}\right)\right]^{1 / \alpha}\left[\ln U_{3}\right]^{1 / \alpha-1}},
$$




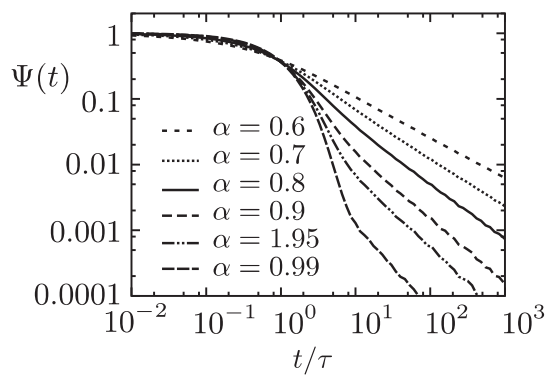

a

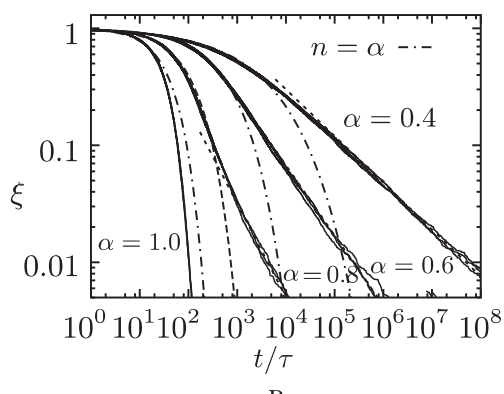

B

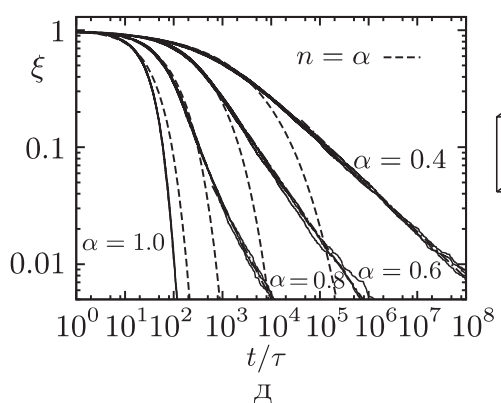

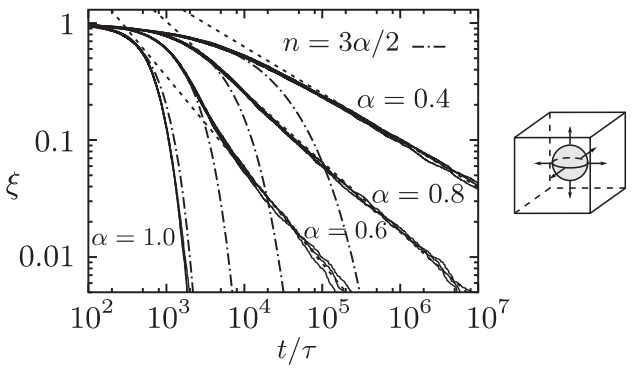

б

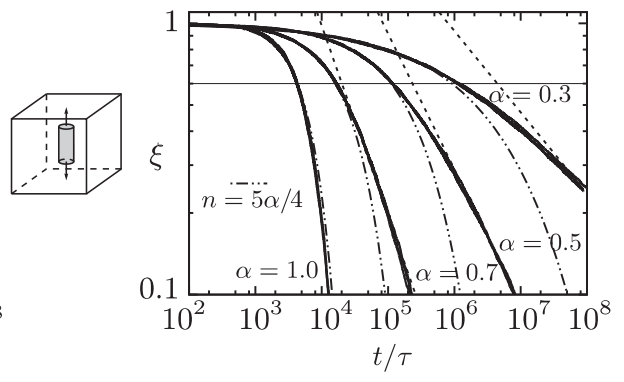

$\Gamma$

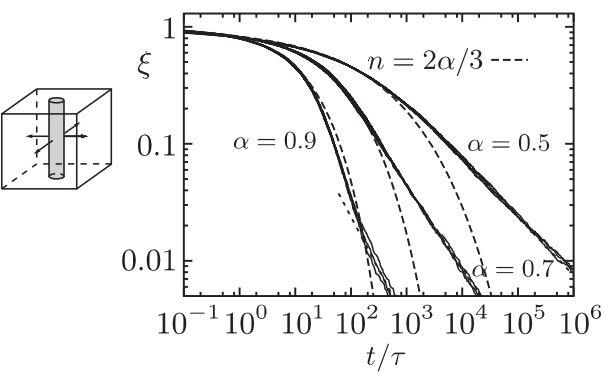

e

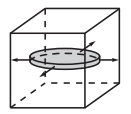

Рис. 3. Результаты моделирования методом Монте-Карло кинетики роста преципитатов различной формы в рамках субдиффузионного обобщения подхода Хэма: дополнительная функция распределения времен ожидания (а) и временные зависимости убывания степени пересыщения в случае различной формы преципитатов и направления роста (б-е) - сферические выделения (изотропный рост) (б), цилиндрические (рост по высоте) (в), дискообразные (увеличение радиуса) (г), стержневидные (увеличение радиуса) (д), плоские (е).

где $U_{1}, U_{2}$ и $U_{3}$ - независимые равномерно распределенные на интервале $(0,1)$ случайные числа. При $\alpha=1$ этот алгоритм сводится к известному алгоритму моделирования случайной величины $T \stackrel{\mathrm{d}}{=}|\ln U| / \mu$ с экспоненциальным распределением. Распределение времен ожидания (28) представлено на рис. За. Для всех значений $0<\alpha<1$ функция $\Psi(t)$ характеризуется степенной асимптотикой $t^{-\alpha}$ при $t \rightarrow \infty$. При $\alpha \rightarrow 1$ мы имеем $\Psi(t) \rightarrow e^{-\mu t}$. 
Набор частиц ранжировался по возрастанию $T_{i}$. Номер 1 присваивался частице с наименьшим временем ожидания и т.д. Естественно, из ловушки выходит раньше всего частица с наименьшим $T$, т. е. для частицы с номером 1 разыгрывается перескок в один из соседних свободных узлов. Если частица попадает в узел, соседствующий с выделением новой фазы, она сама становится частью его и выбывает из ансамбля "свободных" частиц. Фиксируется время присоединения этой частицы. Порядок всех оставшихся частиц уменьшается на единицу. Радиус кластера пересчитывается согласно его плотности. Условия присоединения, конечно, могут быть модифицированы. Нас в первую очередь интересовало влияние субдиффузии на кинетику преципитации. Если узел, в который попадает частица, не соседствует с выделением новой фазы, частица снова локализуется, и разыгрывается время ожидания $\Delta T_{1}$, которое добавляется к текущему $T_{1}$. Теперь частица с приписанным временем $T_{1}+\Delta T_{1}$ и новыми координатами занимает положенное место $k$ в ранжированном ряде частиц, но прежде все частицы с номером, не превосходящим $k$, уменьшают свой номер на единицу. Процесс заканчивается, когда все частицы становятся частью выделения новой фазы.

Важное достоинство алгоритма, например, по сравнению со схемой, в которой разыгрываются темпы делокализации, заключается в том, что скорость счета напрямую не зависит от времени роста выделения новой фазы и, следовательно, от $\alpha$.

На рис. 3б представлены результаты численных расчетов кинетики роста сферических выделений новой фазы для различных значений $\alpha$. Отношение плотностей $\rho_{\mathrm{c}} / \rho_{0}=6.4$, число частиц $N_{\mathrm{p}}=10^{4}$. Масштабный параметр по времени подбирался для каждого случая $\alpha$, чтобы представить кривые на одном графике. "Хвосты" этих зависимостей сравниваются со степенными законами (24), начальные участки сравниваются с зависимостями (23). Таким образом, результаты численных расчетов согласуются с аналитическими решениями. Для каждого значения $\alpha$ представлены результаты для трех реализаций ячейки. На рис. 3в-3е представлены результаты моделирования для различных форм преципитатов и направлений роста, на начальной стадии кривые аппроксимировались зависимостями $e^{-A t^{n \alpha}}$ (значения $n$ указаны на рисунке), на финальной - зависимостью $t^{-\alpha}$.

\section{5. СУБДИФФУЗИОННОЕ ОБОБЩЕНИЕ МОДЕЛИ ААРОНА-КОТЛЕРА}

В работах [6], [7] для описания эволюции изолированного сферического преципитата в бесконечной матрице ставится следующая граничная задача Стефана: необходимо решить уравнение нормальной изотропной диффузии с граничными условиями

$$
\begin{gathered}
\left.\rho(r, t)\right|_{r=R}=\rho_{\mathrm{I}},\left.\quad \rho(r, t)\right|_{r \rightarrow \infty}=\rho_{\mathrm{M}}, \\
\left(\rho_{\mathrm{p}}-\rho_{\mathrm{I}}\right) \frac{d R}{d t}=\left.D \frac{\partial \rho}{\partial r}\right|_{r=R}, \quad t>0,
\end{gathered}
$$

и начальным условием

$$
\left.\rho(r, t)\right|_{t=0}=\rho_{\mathrm{M}}, \quad r>R .
$$


Здесь $\rho_{\mathrm{p}}$ и $\rho_{\mathrm{M}}-$ соответственно концентрации частиц в преципитате и в матрице на бесконечности, $\rho_{\mathrm{I}}-$ равновесная концентрация на поверхности преципитата со стороны матрицы.

Модифицируем эту задачу для случая субдиффузионно-ограниченного распада (или растворения) сферического преципитата. Уравнение изотропной субдиффузии запишем в сферических координатах:

$$
\frac{\partial \rho}{\partial t}=C_{\alpha 0} \mathrm{D}_{t}^{1-\alpha}\left[\frac{\partial^{2} \rho}{\partial r^{2}}+\frac{2}{r} \frac{\partial \rho}{\partial r}\right] .
$$

Граничные условия (29) остаются справедливыми и в субдиффузионном случае, а вот выражение (30) для движения границы согласно соотношениям (11), (12) заменяется уравнением

$$
\left(\rho_{\mathrm{p}}-\rho_{\mathrm{I}}\right) \frac{d R}{d t}=C_{\alpha{ }_{0}} \mathrm{D}_{t}^{1-\alpha}\left[\frac{\partial \rho}{\partial r}\right]_{r=R}
$$

или уравнением (в случае $\rho_{\mathrm{I}}=$ const)

$$
\left(\rho_{\mathrm{p}}-\rho_{\mathrm{I}}\right)_{0} \mathrm{D}_{t}^{\alpha} R=\left.C_{\alpha} \frac{\partial \rho}{\partial r}\right|_{r=R} .
$$

Решим эту задачу для дробно-дифференциального уравнения диффузии в приближении "стационарного интерфейса", предполагающем медленное движение границы преципитата, т. е. выполнение условия $\left|\rho_{\mathrm{M}}-\rho_{\mathrm{I}}\right| \ll \rho_{\mathrm{p}}-\rho_{\mathrm{I}}$. В рамках этого приближения сначала решается диффузионное уравнение с заданными граничными условиями на неподвижной границе $R=$ const для произвольного $R$. Затем после подстановки решения в уравнение (33) находится зависимость $R(t)$. Переходя к функции $u(r, t)=r\left[\rho(r, t)-\rho_{\mathrm{M}}\right]$, получаем уравнение

$$
\frac{\partial u}{\partial t}=C_{\alpha 0} \mathrm{D}_{t}^{1-\alpha} \frac{\partial^{2} u}{\partial r^{2}}
$$

с граничными условиями

$$
\left.u(r, t)\right|_{r=R}=R\left(\rho_{\mathrm{I}}-\rho_{\mathrm{M}}\right),\left.\quad u(r, t)\right|_{r \rightarrow \infty}=0, \quad t>0,
$$

и начальным условием $\left.u(r, t)\right|_{t=0}=0$ при $r>R$. С учетом последнего условия соотношение (35) может быть переписано в виде

$$
{ }_{0} \mathrm{D}_{t}^{\alpha} u=C_{\alpha} \frac{\partial^{2} u}{\partial r^{2}}
$$

его преобразование Лапласа приводит к уравнению

$$
s^{\alpha} \tilde{u}(r, s)-C_{\alpha} \frac{\partial^{2} \tilde{u}(r, s)}{\partial r^{2}}=0 .
$$

Решение этого уравнения с учетом указанных граничных условий имеет вид

$$
\tilde{u}(r, s)=\frac{R\left(\rho_{\mathrm{I}}-\rho_{\mathrm{M}}\right)}{s} \exp \left\{-(r-R) \frac{s^{\alpha / 2}}{\sqrt{C_{\alpha}}}\right\} .
$$


Переходя к трансформанте концентрации, получаем

$$
\tilde{\rho}(r, s)=\frac{\rho_{\mathrm{M}}}{s}+\frac{\tilde{u}(r, s)}{r}=\frac{\rho_{\mathrm{M}}}{s}+\frac{R\left(\rho_{\mathrm{I}}-\rho_{\mathrm{M}}\right)}{r s} \exp \left\{-(r-R) \frac{s^{\alpha / 2}}{\sqrt{C_{\alpha}}}\right\} .
$$

Обратное преобразование Лапласа приводит нас к выражению

$$
\rho(r, t)=\rho_{\mathrm{M}}+\frac{R\left(\rho_{\mathrm{I}}-\rho_{\mathrm{M}}\right)}{r} \int_{0}^{t} d \tau\left(\frac{r-R}{\sqrt{C_{\alpha}}}\right)^{-2 / \alpha} g_{+}^{(\alpha / 2)}\left(\tau\left[\frac{r-R}{\sqrt{C_{\alpha}}}\right]^{-2 / \alpha}\right),
$$

или

$$
\rho(r, t)=\rho_{\mathrm{M}}+\frac{R\left(\rho_{\mathrm{I}}-\rho_{\mathrm{M}}\right)}{r} G_{+}^{(\alpha / 2)}\left(t\left[\frac{r-R}{\sqrt{C_{\alpha}}}\right]^{-2 / \alpha}\right) .
$$

Здесь $g_{+}^{(\gamma)}(t)$ - односторонняя устойчивая плотность с характеристическим показателем $\gamma$ (субординатор), а $G_{+}^{(\gamma)}(t)$ - соответствующая функция распределения [17].

Значение градиента концентрации

$$
\begin{aligned}
\frac{\partial \rho}{\partial r}= & -\frac{R\left(\rho_{\mathrm{I}}-\rho_{\mathrm{M}}\right)}{r^{2}} G_{+}^{(\alpha / 2)}\left(t\left[\frac{r-R}{\sqrt{C_{\alpha}}}\right]^{-2 / \alpha}\right)- \\
& -\frac{2 t}{\alpha \sqrt{C_{\alpha}}}\left(\frac{r-R}{\sqrt{C_{\alpha}}}\right)^{-2 / \alpha-1} \frac{R\left(\rho_{\mathrm{I}}-\rho_{\mathrm{M}}\right)}{r} g_{+}^{(\alpha / 2)}\left(t\left[\frac{r-R}{\sqrt{C_{\alpha}}}\right]^{-2 / \alpha}\right)
\end{aligned}
$$

на поверхности преципитата $r=R$ (с использованием асимптотики для устойчивой плотности распределения [17]) принимает вид

$$
\left.\frac{\partial \rho}{\partial r}\right|_{r \rightarrow R}=\frac{\rho_{\mathrm{M}}-\rho_{\mathrm{I}}}{R}+\frac{t^{-\alpha / 2}\left(\rho_{\mathrm{M}}-\rho_{\mathrm{I}}\right)}{\Gamma(1-\alpha / 2) \sqrt{C_{\alpha}}} .
$$

Подстановка последнего соотношения в уравнение (34) для функции $R(t)$ приводит к уравнению

$$
{ }_{0} \mathrm{D}_{t}^{\alpha} R=C_{\alpha} \frac{\beta(R)}{R}+\sqrt{C_{\alpha}} \frac{\beta(R) t^{-\alpha / 2}}{\Gamma(1-\alpha / 2)}, \quad \beta(R)=\frac{\rho_{\mathrm{M}}-\rho_{\mathrm{I}}(R)}{\rho_{\mathrm{p}}-\rho_{\mathrm{I}}(R)} .
$$

В случае $\alpha=1, C_{1}=D$ мы имеем

$$
\rho(r, t)=\rho_{\mathrm{M}}+\frac{R\left(\rho_{\mathrm{I}}-\rho_{\mathrm{M}}\right)}{r} G_{+}^{(1 / 2)}\left(t\left[\frac{r-R}{\sqrt{D}}\right]^{-2}\right)=\rho_{\mathrm{M}}+\frac{R\left(\rho_{\mathrm{I}}-\rho_{\mathrm{M}}\right)}{r} \operatorname{erfc}\left(\frac{r-R}{2 \sqrt{D t}}\right)
$$

т. е. приходим к классическому результату, справедливому для нормальной диффузии [6]. 
Рассмотрим случай роста сферического выделения новой фазы, управляемого субдиффузией, при условии $\rho_{\mathrm{I}}(R)=\mathrm{const}$, и, соответственно, $\beta=\left(\rho_{\mathrm{M}}-\rho_{\mathrm{I}}\right) /\left(\rho_{\mathrm{p}}-\rho_{\mathrm{I}}\right)=$ const. Решение уравнения (39) ищем в виде

$$
R(t)=\lambda\left(C_{\alpha} t^{\alpha}\right)^{1 / 2}
$$

Подстановка этой функции и дробной производной от нее,

$$
\begin{aligned}
{ }_{0} \mathrm{D}_{t}^{\alpha} R & =\frac{\lambda C_{\alpha}^{1 / 2}}{\Gamma(1-\alpha)} \frac{d}{d t} \int_{0}^{t} d \tau \tau^{\alpha / 2}(t-\tau)^{\alpha}=\frac{(1-\alpha / 2) \lambda C_{\alpha}^{1 / 2}}{\Gamma(1-\alpha)} t^{-\alpha / 2} \mathrm{~B}\left(1+\frac{\alpha}{2} ; 1-\alpha\right)= \\
& =\frac{\alpha \Gamma(\alpha / 2)}{\Gamma(1-\alpha / 2)} \lambda C_{\alpha}^{1 / 2} t^{-\alpha / 2}
\end{aligned}
$$

в формулу (39) приводит к квадратному уравнению

$$
\alpha \Gamma\left(\frac{\alpha}{2}\right) \lambda^{2}-\beta \lambda-\Gamma\left(1-\frac{\alpha}{2}\right) \beta=0,
$$

положительный корень которого есть

$$
\lambda=\frac{\beta+\sqrt{\beta^{2}+4 \beta \pi \alpha / \sin \pi \alpha}}{2 \alpha \Gamma(\alpha / 2)} .
$$

Таким образом, выражение (40) дает закон субдиффузионно-ограниченного роста сферического преципитата при постоянном $\beta$ и обобщает выражение $R(t) \propto t^{1 / 2}$, полученное для нормальной диффузии [6].

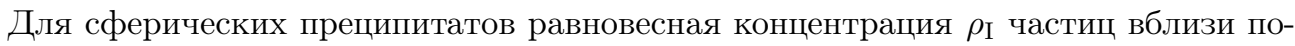
верхности преципитата со стороны матрицы задается выражением Гиббса-Томсона

$$
\rho_{\mathrm{I}}=\rho_{\mathrm{I}}^{\infty} \exp \left(\frac{A}{k T R}\right)
$$

Здесь $A$ - константа, зависящая от состава. Для меди в $\alpha$-железе $A=672.51 k[46]$.

На рис. 4 представлены результаты моделирования методом Монте-Карло растворения сферического преципитата при нормальной диффузии примесей с учетом эффекта Гиббса-Томсона. Уравнение (39) для функции $R(t)$ можно также решить одним из численных методов, специально разработанных для дифференциальных уравнений дробного порядка [39]-[42].

Результаты решения с помощью конечно-разностного метода (после аппроксимации дробной производной Римана-Лиувилля производной Грюнвальда-Летникова) представлены на рис. 5. Кинетика растворения и роста сферических выделений при уменьшении $\alpha$ замедляется (становится более пологой). В случае роста кривые в асимптотике больших времен стремятся к зависимости $t^{\alpha / 2}$. В пределе $\alpha \rightarrow 1$ решения, найденные с помощью специального численного алгоритма для дробных 

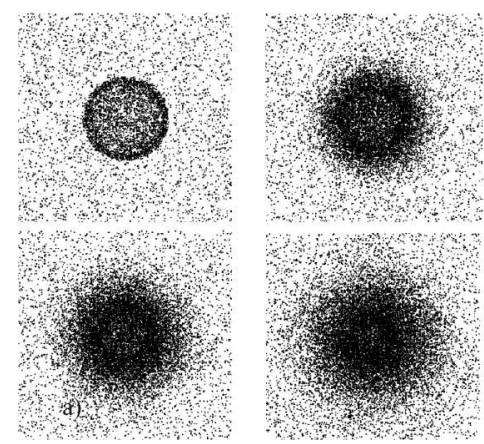

a

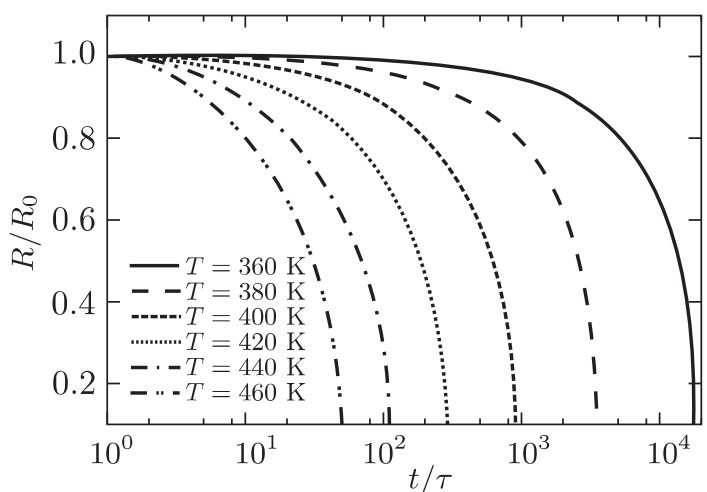

б

Рис. 4. Результаты моделирования методом Монте-Карло растворения сферического преципитата при нормальной диффузии примесей с учетом эффекта Гиббса-Томсона: эволюция распределения примесей вблизи преципитата в проекции на плоскость $x y(\mathrm{a})$; кинетика диффузионно-ограниченного растворения при различных температурах (б).

$R_{0}=6 \mathrm{HM}, C_{\mathrm{M}}=0.127 C_{1}, T=380 \mathrm{~K}$

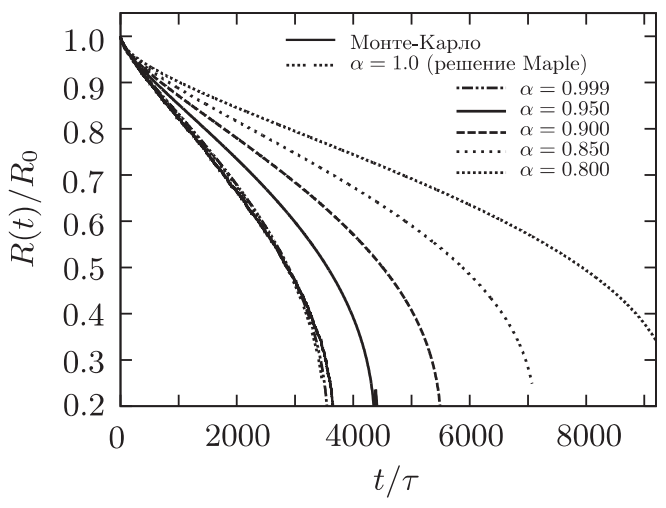

a

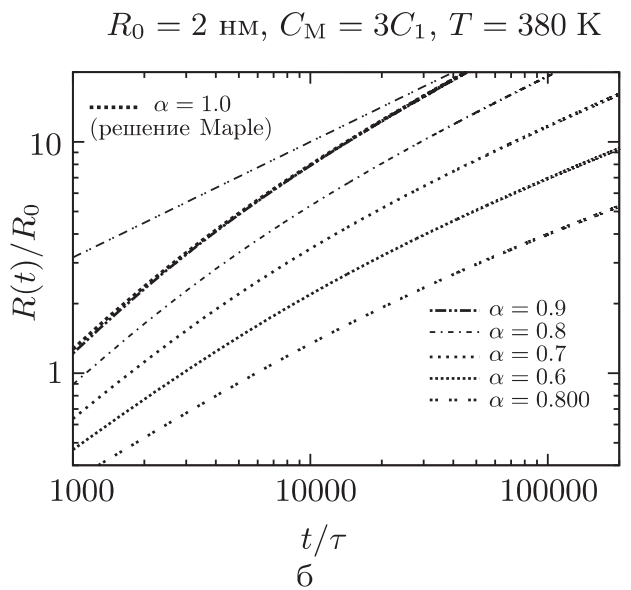

б

Рис. 5. Кинетика субдиффузионного растворения (а) и роста (б) сферических выделений для различных показателей аномальной диффузии $\alpha$, рассчитанная в рамках дробно-дифференциального обобщения модели Аарона-Котлера.

значений $\alpha$, приближаются к результату, рассчитанному стандартным конечно-разностным методом для случая $\alpha=1$, который, в свою очередь, близок к кривой, найденной с помощью моделирования методом Монте-Карло. Это указывает на выполнение принципа соответствия и применимость приближения стационарного интерфейса для выбранных параметров. 


\section{6. ЗАКЛЮЧЕНИЕ}

В предложенной работе рассмотрена кинетика субдиффузионно-ограниченного роста и растворения частиц новой фазы в твердых растворах с помощью современного подхода, основанного на кинетических уравнениях с производными дробного порядка и соответствующих алгоритмах моделирования методом Монте-Карло. Рассматриваются обобщения известных моделей преципитации Хэма [2], [3] и Аарона-Котлера [6], [7]. Полученные в рамках обобщений решения согласуются с результатами моделирования методом Монте-Карло. С помощью моделирования рассмотрена кинетика роста преципитатов различной формы: сферической, дискообразной, цилиндрической, стержневидной и некоторых других. При росте преципитатов начальный участок кривой спада пересыщения описывается зависимостью $\sim e^{-k t^{n \alpha}}$, на больших временах асимптотика степенная с показателем $-\alpha$ для всех рассмотренных форм. В обобщенной модели Аарона-Котлера аналитические результаты получены главным образом в приближении стационарного интерфейса. Профили частиц в матрице выражены через устойчивые распределения, являющиеся обобщением гауссова распределения. Без учета эффекта Гиббса-Томсона в субдиффузионных обобщениях моделей Хэма и Аарона-Котлера получено, что кинетика роста сферических кластеров описывается степенным законом $\propto t^{\alpha / 2}$. С учетом эффекта Гиббса-Томсона временная зависимость радиуса оказывается более крутой на начальных стадиях роста, а в асимптотике больших времен выходит на степенную зависимость $t^{\alpha / 2}$.

Полученные результаты могут оказаться полезными при анализе экспериментальных и модельных данных по преципитации. В частности, имеющиеся данные для системы $\mathrm{Cu}-\mathrm{Fe}$ [47]-[51] свидетельствуют о степенном законе роста сферических преципитатов меди в $\alpha$-железе с показателем 0.4 , что находится в противоречии с результатами упомянутых нормально-диффузионных моделей и классической моделью коалесценции. Данные из работы [52] по растворению нанопреципитатов кремния в аллюминиевой матрице при температурах отжига $500-560{ }^{\circ} \mathrm{C}$ показывают замедленное поведение по сравнению с предсказываемым классической диффузионной моделью растворения сферических преципитатов [53]. В связи с этим мы планируем посвятить следующую работу детальному анализу имеющихся экспериментальных данных в рамках предложенных дробно-дифференциальных моделей.

Благодарности. Настоящая работа выполнена при поддержке РФФИ (грант № 15-01-99674) и Министерства образования и науки РФ в рамках государственного задания 2014/296 (1643, 3.1862.2014K).

\section{Список литературы}

[1] В. В. Слезов, В. В. Сагалович, УФН, 151:1 (1987), 67-104.

[2] F. S. Ham, J. Phys. Chem. Solids, 6:4 (1958), 335-351.

[3] F. S. Ham, J. Appl. Phys., 30:10 (1959), 1518-1525.

[4] H. B. Aaron, Metal Sci., 2:1 (1968), 192-193.

[5] M. J. Whelan, Metal Sci., 3:1 (1969), 95-97.

[6] H. B. Aaron, D. Fainstein, G. R. Kotler, J. Appl. Phys., 41:11 (1970), 4404-4410.

[7] H. B. Aaron, G. R. Kotler, Metal. Mater. Trans. B, 1971, № 2, 393-408.

[8] А. В. Гапонцев, В. В. Кондратьев, УФН, 173:10 (2003), 1107-1129. 
[9] В. Л. Гапонцев, И. К. Разумов, Ю. Н. Горностырев, А. Е. Ермаков, В. В. Кондратьев, $\Phi M M, \mathbf{9 9 : 4}(2005), 26-37$.

[10] И. М. Лифшиц, В. В. Слёзов, ЖЭТФ, 35:2(8) (1958), 479-492.

[11] S. Liu, L. Bönig, J. Detch, H. Metiu, Phys. Rev. Lett., 74:22 (1995), 4495-4498.

[12] J. P. Bouchaud, A. Georges, Phys. Rep., 195:4-5 (1990), 127-293.

[13] M. B. Isichenko, Rev. Modern Phys., 64:4 (1992), 961-1043.

[14] R. Metzler, J. Klafter, Phys. Rep., 339:1 (2000), 1-77.

[15] Y. Gefen, A. Aharony, S. Alexander, Phys. Rev. Lett., 50:1 (1983), 77-80.

[16] S. B. Yuste, K. Lindenberg, Chem. Phys., 284:1 (2002), 169-180.

[17] В. В. Учайкин, УФН, 173:8 (2003), 847-876.

[18] V. V. Uchaikin, R. T. Sibatov, Fractional Kinetics in Solids: Anomalous Charge Transport in Semiconductors, Dielectrics and Nanosystems, CRC Press, Boca Raton, FL, 2013.

[19] Р. Т. Сибатов, В.В.Учайкин, УФН, 179:10 (2009), 1079-1104.

[20] Р. Т. Сибатов, В.В.Учайкин, ФТП, 41:3 (2007), 346-351.

[21] В. В. Учайкин, Р. Т. Сибатов, Писъма в ЖЭТФ, 86:8 (2007), 584-588.

[22] В. П. Шкилев, ЖЭТФ, 143:4 (2013), 804-812.

[23] P. Paradisi, R. Cesari, F. Mainardi, F. Tampieri, Physica A: Stat. Mech. Appl., 293:1-2 (2001), 130-142.

[24] V. M. Kenkre, E. W. Montroll, M. F. Shlesinger, J. Statist. Phys., 9:1 (1973), 45-50.

[25] M. F. Shlesinger, J. Statist. Phys., 10:5 (1974), 421-434.

[26] H. Scher, E. W. Montroll, Phys. Rev. B, 12:6 (1975), 2455-2477.

[27] J. Klafter, R. Silbey, Phys. Rev. Lett., 44:2 (1980), 55-58.

[28] A. Blumen, J. Klafter, G. Zumofen, Phys. Rev. B, 27:6 (1983), 3429-3435.

[29] R. Gorenflo, F. Mainardi, A. Vivoli, Chaos, Solitons 8 Fractals, 34:1 (2007), 87-103, arXiv: cond-mat/0701126.

[30] E. W. Montroll, G. H. Weiss, J. Math. Phys., 6:2 (1965), 167-181.

[31] В. В. Учайкин, Метод дробных производных, Артишок, Ульяновск, 2008.

[32] K. B. Oldham, J. Spanier, The Fractional Calculus, Mathematics in Science and Engineering, 111, Academic Press, New York, 1974.

[33] I. Podlubny, Fractional Differential Equations, Mathematics in Science and Engineering, 198, Academic Press, San Diego, CA, 1999.

[34] J. W. Cahn, J. E. Hilliard, The J. Chem. Phys., 28:2 (1958), 258-267.

[35] A. Milchev, D. W. Heermann, K. Binder, Acta Metallurgica, 36:2 (1988), 377-383.

[36] E. Sander, T. Wanner, J. Statist. Phys., 95:5-6 (1999), 925-948.

[37] A. Cortis, C. Gallo, H. Scher, B. Berkowitz, Water Resour. Res., 40:4 (2004), W04209, $16 \mathrm{pp}$.

[38] В. В. Светухин, Р. Т. Сибатов, ЖЭТФ, 147:4 (2015), 782-792.

[39] K. Diethelm, N. J. Ford, J. Math. Anal. Appl., 265:2 (2002), 229-248.

[40] K. Diethelm, N. J. Ford, A. D. Freed, Numer. Algor., 36:1 (2004), 31-52.

[41] K. Diethelm, N. J. Ford, A. D. Freed, Yu. Luchko, Comput. Methods Appl. Mech. Engrg., 194:6-8 (2005), 743-773.

[42] C. Li, F. Zeng, Numer. Funct. Anal. Optim., 34:2 (2013), 149-179.

[43] F. Mainardi, R. Gorenflo, J. Comput. Appl. Math., 118:1-2 (2000), 283-299.

[44] V. V. Uchaikin, R. T. Sibatov, Russ. J. Numer. Anal. Math. Model., 23:3 (2008), 283-297.

[45] V. V. Uchaikin, D. O. Cahoy, R. T. Sibatov, Internat. J. Bifurcation Chaos Appl. Sci. Engrg., 18:9 (2008), 2717-2725.

[46] G. Salje, M. Feller-Kniepmeier, J. Appl. Phys., 48:5 (1977), 1833-1839. 
[47] R. Kampmann, R. Wagner, "Phase transformations in Fe-Cu-Alloys-SANS-experiments and theory", Atomic Transport and Defects in Metals by Neutron Scattering (Jülich, Fed. Rep. of Germany, October 2-4, 1985), Springer Proceedings in Physics, 10, eds. C. Janot, W. Petry, D. Richter, Springer, New York, 1986, 73-77.

[48] M. H. Mathon, A. Barbu, F. Dunstetter, F. Maury, N. Lorenzelli, C. H. De Novion, J. Nucl. Mater., 245:2 (1997), 224-237.

[49] S.R. Goodman, S.S. Brenner, J.R. Low, Metallurgical Transactions, 4:10 (1973), 2371-2378.

[50] M. Perez, F. Perrard, V. Massardier, X. Kleber, A. Deschamps, H. De Monestrol, P. Pareige, G. Covarel, Philosophical Magazine, 85:20 (2005), 2197-2210.

[51] F. Soisson, C.-C. Fu, Phys. Rev. B, 76:21 (2007), 214102, 12 pp.

[52] U. H. Tundal, N. Ryum, Metal. Trans. A, 23:2 (1992), 445-449.

[53] T. L. Durbin, Modeling dissolution in aluminum alloys, $\mathrm{PhD}$ thesis, Mechanical Engineering, Georgia Institute of Technology, Atlanta, GA, 2005. 